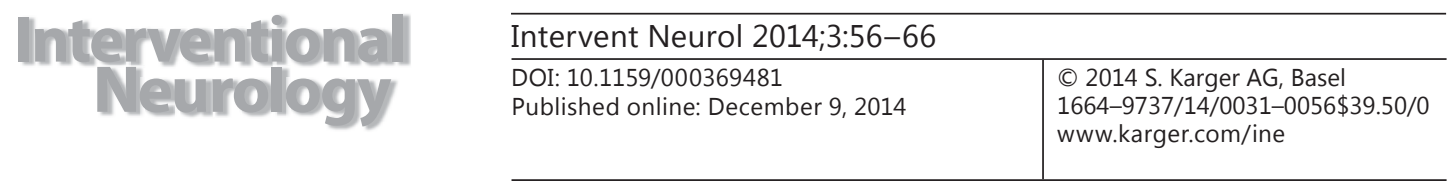

\title{
Aggressive Medical Care in Young Chinese Patients with Ischemic Stroke of Undetermined Etiology: A Retrospective Study
}

\author{
Ling Liu Fang Yang Min Li Huajuan Hou Qin Yin Renliang Zhang \\ Department of Neurology, Jinling Hospital, Nanjing University School of Medicine, \\ Nanjing, PR China
}

\section{Key Words}

Medical care $\cdot$ Undetermined etiology $\cdot$ Young stroke patients

\begin{abstract}
Objectives: This study aimed to investigate the clinical and angiographic characteristics of ischemic stroke of undetermined etiology in young Chinese adults and to observe the effects of medication on their long-term outcomes. Methods: A total of 179 consecutive young patients with ischemic stroke of undetermined etiology were retrospectively analyzed for clinical and angiographic characteristics, laboratory tests, the choice of drug treatment, and followup outcomes. Any predictive power for recurrent stroke and new lesions or aggravated stenosis was analyzed. Results: 170 patients were clinically followed up for a median of 25 months (range, 4-92), and 65 patients with 114 lesions had angiographic follow-up for a median of 7 months. A total of 53 patients were enrolled in a routine medical management (RMM) group, and 117 were treated with corticosteroids plus RMM (aggressive medical management, AMM). Kaplan-Meier survival analysis revealed that differences in the 2-year cumulative stroke-free rate and in the 18-month cumulative worsening and/or new lesion-free rate between the RMM and AMM groups were significant $(p<0.05)$. Multivariate and Cox regression analyses revealed that the choice of drug therapy and erythrocyte sedimentation rate were associated with recurrent stroke, that AMM was independently associated with a decreased risk of worsening lesion, and that worsening lesion was the only independent predictor of recurrent
\end{abstract}

L.L., F.Y., M.L., and H.H. contributed equally to this work. 
stroke. Conclusion: Compared with RMM, AMM is more efficacious in the prevention of secondary ischemic stroke and progressive arterial lesions among young adults with ischemic stroke of undetermined etiology.

(C) 2014 S. Karger AG, Basel

\section{Introduction}

Ischemic strokes occurring in patients aged $<45$ years have been considered relatively rare events, accounting for up to $12 \%$ of all ischemic strokes [1]. Compared to old patients, young stroke patients have more diverse etiologies, and the etiologic frequency of ischemic stroke in young patients differs around the world [2,3]. The proportion of ischemic strokes of undetermined etiology is 30-70\% among young patients in different countries and regions, which is significantly higher than in elderly stroke patients [4].

Although stroke occurs at a relatively lower annual incidence rate in young adults, it has a relatively higher public health impact because of direct socioeconomic and associated indirect costs $[5,6]$. This phenomenon is especially striking in China, as it is a developing country with a population of $>1.3$ billion and an uneven development of regional economies. Until now, published studies on young adults suffering from stroke mainly focused on its clinical subtypes, the distribution of stroke etiologies, prognosis, and associated cerebrovascular disease risk factors [7] and rarely referred to its medication and angiographic characteristics. In the present study, we attempted to retrospectively review the incidence of ischemic stroke of undetermined etiology among young Chinese patients (aged 18-45 years) as well as their clinical and angiographic characteristics. Meanwhile, we compared the efficacy of aggressive (AMM) and routine medical management (RMM) in clinical and angiographic outcomes.

\section{Materials and Methods}

\section{Patients}

This study was approved by the ethics committee of our institution, and written informed consent was obtained from all patients and their families.

The Nanjing Stroke Registry Program (NSRP) [8] is a program conducted in mainland China, which contains a variety of information including demographic, clinical, and imaging data as well as laboratory tests. Ischemic stroke patients aged <45 years were recruited from the NSRP between March 2005 and March 2012, and their medical records were reviewed to exclude those who presented with determined etiologies such as atherosclerosis, cardiac disease, etc. Finally, a total of 179 eligible young patients with ischemic stroke were enrolled, and their baseline data, drug management, and angiographic and clinical follow-up outcomes were retrospectively collected and analyzed.

\section{Medication}

All eligible patients received RMM as follows: (1) antiplatelet therapy (aspirin $100 \mathrm{mg} /$ day, clopidogrel $75 \mathrm{mg} /$ day, or a combination of both); (2) a cholesterol-lowering agent (statins such as atorvastatin or rosuvastatin) unless there were drug contraindications, and (3) intravenous administration of penicillin G (9.614.4 million units per day for 10-14 days) or oral azithromycin therapy ( $0.5 \mathrm{~g}$ per day for 5 days) for patients allergic to penicillin.

The participants who provided written informed consent then received corticosteroid therapy (AMM) as follows: high-dose methylprednisolone pulse therapy (1,000 mg/day i.v.) for 5 days followed by oral prednisone sequential therapy ( $1 \mathrm{mg} / \mathrm{kg} /$ day) for at least 3 months. If clinical symptoms improved and no stroke recurrence was noted, prednisone was continued on an alternate day regimen and was ultimately tapered to $20 \mathrm{mg} /$ day and stopped within 3 months. At the same time, participants were treated with gastric acid suppressors, potassium, and calcium. During the course of steroid therapy, if transient ischemic attack (TIA) 
or ischemic stroke recurred, or if worsening stenosis or a new lesion was detected, patients in the AMM group were encouraged to start taking immunosuppressive medications such as azathioprine or cyclophosphamide, whereas patients in the RMM group were encouraged to undergo corticosteroid treatment.

\section{Laboratory Assays and Routine Tests}

The following laboratory examinations were performed in all patients: complete blood counts, clinical chemistry parameters, thrombophilia markers including proteins C and S and antithrombin III, homocysteine, lupus anticoagulant and anticardiolipin antibodies, antinuclear antibody spectrum, antineutrophil cytoplasmic antibodies, serum immunoglobulin assay, rheumatoid factor, antistreptolysin 0 (ASO), complement 3 (C3), erythrocyte sedimentation rate (ESR), and C reactive protein (CRP).

All participants received routine tests including a chest X-ray, various neuroimaging (brain CT or MRI scan and angiography) and cardiac tests such as electrocardiography and contrast and/or transthoracic echocardiogram and/or transesophageal echocardiogram performed to exclude certain cryptogenic heart diseases such as patent foramen ovale.

\section{Angiographic Procedure}

Digital subtraction angiography (DSA) included intra- and extracranial portions of bilateral carotid and vertebrobasilar systems. According to a previous report [9], the angiographic procedure was performed on a DynaCT angiography machine (AXIOM Artis dTA; Siemens Healthcare, Erlangen, Germany) at the interventional center of our department. Briefly, under local anesthesia, a 5-Fr cerebral guiding catheter (Envoy; Cordis, Fremont, Calif., USA, or Guider Soft Tip XP; Boston Scientific, Natick, Mass., USA) was passed into the common or internal carotid artery (ICA) and the subclavian or vertebral artery through a 5-Fr femoral arterial sheath. Baseline angiography was performed to assess all vascular lesions. Each angiogram was reviewed by a neuroradiologist and a neurologist. The stenosis rate of the extracranial vessels was calculated according to the North American Symptomatic Carotid Endarterectomy Trial criteria, and the stenosis degree of the intracranial arteries was evaluated using the WASID technique. Mild, moderate, and severe stenosis was defined as 30-49, 50-69, and 70-99\% diameter stenosis, respectively. All the 179 patients underwent DSA assessment, and in 95 of them a total of 169 stenotic arteries $(\geq 30 \%)$ were found.

\section{Clinical and Angiographic Follow-Up}

All participants were scheduled for a clinical follow-up or a complete telephone interview at 1, 3, 6, and 12 months and yearly thereafter if they had no recurrence. All patients with significant arterial stenosis were scheduled for an angiographic follow-up at 6 months after the first registration, or earlier in the case of either recurrent TIA or ischemic stroke. Patients with recurrence of stroke or TIA required DSA reevaluation. The degree of stenosis on CT or MR angiography was based on the interpretations by the staff neuroradiologists. In patients whose noninvasive imaging findings were ambiguous or suggested progressive stenosis, DSA was performed. Compared to the initial angiography, a target lesion having an increase in the degree of stenosis of $\geq 10 \%$ or developing total occlusion was regarded as worsening stenosis, a lesion with a decrease in the degree of stenosis of $\geq 20 \%$ or total disappearance was defined as lessening lesion, and a newly verified arterial stenosis $\geq 30 \%$ was defined as a new lesion.

\section{End Points}

The main end points were composite clinical outcome events including nonfatal stroke and death from all causes, and the secondary end points were angiographic outcomes including worsening stenosis and new lesions. Recurrent ischemic symptoms were defined as new episodes of TIA or acute ischemic stroke.

\section{Data Analysis}

Continuous data are expressed as mean \pm standard deviation (SD), and categorical data are presented as numbers and percentages. A $\chi^{2}$ test and one-way ANOVA analysis were performed for multiple comparisons when appropriate. Kaplan-Meier analysis and a log-rank test were performed to detect the differences between the two groups for the time to any neurological event (ischemic stroke and/or TIA) and lesion change. By employing stepwise regression analysis, all variables were investigated to screen for those factors which were related to recurrent stroke and progressive original stenosis or new lesions. The entry and removal probabilities of the stepwise selection were 0.05 and 0.1 , respectively. A p value $<0.05$ was considered statistically significant. All analyses were performed with SPSS version 15.0 (SPSS Inc., Chicago, Ill., USA). 
Table 1. Patient characteristics

\begin{tabular}{|c|c|c|c|}
\hline Characteristics & $\begin{array}{l}\text { Total } \\
(n=179)\end{array}$ & $\begin{array}{l}\text { No DSA follow-up } \\
(\mathrm{n}=114)\end{array}$ & $\begin{array}{l}\text { DSA follow-up } \\
(n=65)\end{array}$ \\
\hline Age, years & $37.6 \pm 6.2$ & $37.0 \pm 6.4$ & $38.5 \pm 5.4$ \\
\hline Male sex & $34(74.9)$ & $85(75.6)$ & $49(75.4)$ \\
\hline \multicolumn{4}{|l|}{ Follow-up, months } \\
\hline Median (range) & $24(0-92)$ & $24(0-85)$ & $24(6-92)$ \\
\hline Mean \pm SD & $31.5 \pm 24.2$ & $31.5 \pm 24.5$ & $31.4 \pm 23.8$ \\
\hline \multicolumn{4}{|l|}{ Blood examination } \\
\hline $\operatorname{ESR}(>10 \mathrm{~mm} / \mathrm{h})$ & $34(19.0)$ & $23(20.2)$ & $11(16.9)$ \\
\hline $\mathrm{C} 3$ & $30(16.3)$ & $18(15.8)$ & $12(18.5)$ \\
\hline $\mathrm{CRP}(>15 \mathrm{mg} / \mathrm{l})$ & $41(22.9)$ & $24(21.1)$ & $17(26.2)$ \\
\hline IgM & $14(7.8)$ & $7(6.1)$ & $7(10.8)$ \\
\hline $\operatorname{IgA}$ & $19(10.6)$ & $11(9.6)$ & $8(12.3)$ \\
\hline $\operatorname{IgG}$ & $38(21.2)$ & $23(20.2)$ & $15(23.1)$ \\
\hline ASO (>20 IU/ml) & $24(13.4)$ & $16(14.0)$ & $8(12.3)$ \\
\hline \multicolumn{4}{|l|}{ Qualifying events } \\
\hline TIA & $40(22.3)$ & $28(24.6)$ & $12(18.5)$ \\
\hline Ischemic stroke & 139 (77.7) & $86(75.4)$ & $53(81.5)$ \\
\hline No stenosis & $84(46.9)$ & $84(73.7)$ & $0(100)$ \\
\hline Lesions, $\mathrm{n}$ & 162 & 48 & 114 \\
\hline \multicolumn{4}{|l|}{ Lesion location } \\
\hline Anterior circulation & 113 & & \\
\hline Extracranial ICA & $47(29.0)$ & $14(29.2)$ & $33(28.9)$ \\
\hline Intracranial ICA & $17(10.5)$ & $4(8.3)$ & $13(11.4)$ \\
\hline Middle cerebral artery & $45(27.8)$ & $11(22.9)$ & $34(29.8)$ \\
\hline Anterior cerebral artery & $4(2.5)$ & $2(4.2)$ & $2(1.8)$ \\
\hline Posterior circulation & 49 & 46 & \\
\hline Posterior cerebral artery & $4(2.5)$ & $2(4.2)$ & $2(1.8)$ \\
\hline Basilar artery & $5(3.1)$ & $2(4.2)$ & $3(2.6)$ \\
\hline Vertebral artery & $40(24.7)$ & $13(27.1)$ & $27(23.7)$ \\
\hline Mean degree of stenosis, $\pm \mathrm{SD}^{\mathrm{a}}$ & $78.37 \pm 12.07$ & $79.09 \pm 12.07$ & $76.67 \pm 12.00$ \\
\hline \multicolumn{4}{|l|}{ Treatment } \\
\hline RMM & $55(30.7)$ & $38(33.3)$ & $17(26.2)$ \\
\hline AMM & $124(69.3)$ & $76(66.7)$ & $48(73.8)$ \\
\hline
\end{tabular}

Data are expressed as numbers with percentages in parentheses unless otherwise indicated.

a The degree of stenosis was determined by the WASID method.

\section{Results}

\section{Baseline Characteristics}

Between March 2005 and March 2012, data of 458 consecutive young patients with ischemic stroke (aged 18-45 years) were extracted from the NSRP, and 179 (39.1\%) of them were diagnosed with ischemia of undetermined etiology. The baseline patient characteristics are presented in table 1. The mean age of all participants was $37.6 \pm 6.2$ years, and 134 $(74.9 \%)$ of the patients were males. The qualifying events before enrolment were cerebral infarction $(77.7 \%)$ and TIA (22.3\%). A total of $55(30.7 \%)$ patients were assigned to the RMM group and $124(69.3 \%)$ to the AMM group.

Blood examination was normal in the majority of the patients; only some had increased serum levels of IgG (20.2\%), IgM (7.3\%), and IgA (10.1\%), ESR (18.4\%), CRP (22.9\%), and ASO (12.8\%), or decreased C3 levels (15.6\%). A total of $84(46.9 \%)$ patients had no DSA- 
Liu et al.: Aggressive Medical Care in Young Chinese Patients with Ischemic Stroke of

Undetermined Etiology: A Retrospective Study

Table 2. Clinical and angiographic outcomes during follow-up

\begin{tabular}{|c|c|c|c|c|c|c|}
\hline \multirow[t]{2}{*}{ End points } & \multirow{2}{*}{$\begin{array}{l}\text { RMM } \\
(n=53)\end{array}$} & \multirow{2}{*}{$\begin{array}{l}\text { AMM } \\
(n=117)\end{array}$} & \multicolumn{4}{|l|}{ HR } \\
\hline & & & unadjusted & $\mathrm{p}$ & adjusted & $\mathrm{p}$ \\
\hline \multicolumn{7}{|l|}{ Clinical follow-up, months } \\
\hline Median (range) & $22(4-85)$ & $31(6-92)$ & $\begin{array}{l}-6.61345 \pm 3.90507 \\
(-14.32279-1.09589)^{a}\end{array}$ & 0.092 & & \\
\hline Mean \pm SD & $28.6 \pm 22.7$ & $35.2 \pm 24.0$ & & & & \\
\hline Mean age $\pm S D$, years & $39.0 \pm 5.5$ & $37.1 \pm 6.2$ & $\begin{array}{c}-1.93162 \pm 0.98747 \\
(-0.1783-3.88107)^{\mathrm{a}}\end{array}$ & 0.052 & & \\
\hline \multicolumn{7}{|l|}{ Clinical outcomes } \\
\hline Recurrence & $10(18.9)$ & $2(1.7)$ & $0.082(0.018-0.375)$ & 0.001 & $0.04(0.008-0.211)$ & 0.000 \\
\hline Angiographic outcomes & 43 & 71 & $0.211(0.077-0.578)$ & 0.002 & $0.160(0.053-0.485)$ & 0.001 \\
\hline Progression & 9 (20.9) & $4(5.6)$ & & & & \\
\hline Improvement & $10(23.3)$ & $44(62.0)$ & & & & \\
\hline No change & $17(39.5)$ & $22(31.0)$ & & & & \\
\hline New lesion & $7(16.3)$ & $1(1.4)$ & & & & \\
\hline \multicolumn{7}{|c|}{ DSA follow-up time, months } \\
\hline Median (range) & $8.0(4.5-20)$ & $7(4-18)$ & $\begin{array}{c}-0.31952 \pm 0.580994 \\
(-0.83164 \text { to } 1.47068)^{a}\end{array}$ & 0.583 & & \\
\hline Mean \pm SD & $8.2 \pm 3.4$ & $7.9 \pm 2.8$ & & & & \\
\hline
\end{tabular}

Values are presented as numbers with percentages in parentheses unless otherwise indicated. Recurrence: cerebral infarction or TIA. Adjusted: adjusted by all the variables presented in table 1 using Cox proportional hazard analyses.

${ }^{a}$ t value $=$ mean \pm SE with $95 \% \mathrm{CI}$ in parentheses .

verified intra- or extracranial artery stenosis (degree of stenosis $<30 \%$ ). We found a total of 162 significant lesions (degree of stenosis $\geq 30 \%)$ in 95 patients: $113(69.8 \%)$ located in the anterior circulation and $49(30.2 \%)$ in the posterior circulation. The mean baseline degree of stenosis was $76.78 \pm 14.44 \%$ (table 1 ). The baseline demographic, laboratory, clinical, and lesional characteristics (location of the lesion and stenosis rate) were comparable between the two medical treatment groups (data not shown).

\section{Clinical and Angiographic Outcomes during Follow-Up}

Of the 179 patients, 9 (5\%; 2 RMM and 7 AMM cases) were lost to follow-up after discharge. Their baseline variables were not different from those of the patients who were followed up (data not shown). Thus, 170 patients could be followed up clinically: 53 RMM and 117 AMM cases. The mean and median clinical follow-up time was $28.6 \pm 22.7$ and 22 months (range, 4-85) for the RMM group and $35.2 \pm 24.0$ and 31 months (range, 6-92) for the AMM group, respectively (table 2).

Clinical follow-up outcomes are shown in table 2. No fatal complications or deaths occurred in this study. Recurrent neurological events (TIA and cerebral infarctions) occurred in 12 cases: $2(1.7 \%)$ in the AMM group and $10(18.9 \%)$ in the RMM group. The composite stroke rate was $6.7 \%(12 / 179)$. Of these cases, $11(91.7 \%)$ were attributed to worsening original stenosis or new lesions. The 2-year cumulative stroke-free survival rate was $86.3 \pm$ $2.9 \%$ in the whole study population, $71.5 \pm 2.2 \%$ in the RMM group, and $92.6 \pm 2.4 \%$ in the AMM group, and the difference was significant (log-rank test, $\chi^{2}=7.459, p=0.006$ ) (fig. 1a).

A median DSA follow-up of 7 months (range, 4-20) was available for 65 patients $(68.4 \%$ of the 95 patients with significant arterial stenosis) with 114 lesions, and 105 patients with 48 lesions were not followed up by DSA. The baseline characteristics of patients with and 


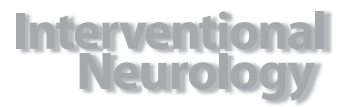

Table 3. Clinical variables at baseline vs. risk of stroke recurrence during clinical follow-up

\begin{tabular}{|c|c|c|c|}
\hline \multicolumn{4}{|c|}{ Intervent Neurol 2014;3:56-66 } \\
\hline \multicolumn{2}{|l|}{ DOI: 10.1159/000369481 } & \multicolumn{2}{|c|}{$\begin{array}{l}\text { (C) } 2014 \text { S. Karger AG, Basel } \\
\text { www.karger.com/ine }\end{array}$} \\
\hline \multicolumn{4}{|c|}{$\begin{array}{l}\text { Liu et al.: Aggressive Medical Care in Young Chinese Patients with Ischemic Stroke of } \\
\text { Undetermined Etiology: A Retrospective Study }\end{array}$} \\
\hline & $\begin{array}{l}\text { Total } \\
(n=170)\end{array}$ & $\begin{array}{l}\text { Recurrence } \\
(n=12)\end{array}$ & $\mathrm{p}$ \\
\hline Mean age \pm SD, years & $37.7 \pm 6.0$ & $39.9 \pm 5.0$ & 0.180 \\
\hline \multicolumn{4}{|l|}{ Sex } \\
\hline Male & $128(75.3)$ & $8(66.7)$ & 0.339 \\
\hline Female & $42(24.7)$ & $4(33.3)$ & \\
\hline \multicolumn{4}{|l|}{ Blood examination } \\
\hline $\operatorname{ESR}(\geq 10 \mathrm{~mm} / \mathrm{h})$ & $29(17.1)$ & $5(41.7)$ & 0.034 \\
\hline $\mathrm{C} 3$ & $30(17.6)$ & $2(16.7)$ & 0.643 \\
\hline ASO ( $\geq 20 \mathrm{IU} / \mathrm{ml})$ & $21(12.4)$ & $1(8.3)$ & 0.548 \\
\hline $\mathrm{CRP}(\geq 15 \mathrm{mg} / \mathrm{l})$ & $39(22.9)$ & $3(25.0)$ & 0.548 \\
\hline $\operatorname{Ig} M$ & $13(7.6)$ & $1(8.3)$ & 0.628 \\
\hline $\operatorname{Ig} A$ & $18(10.6)$ & $1(8.3)$ & 0.630 \\
\hline IgG & $38(22.4)$ & $2(16.7)$ & 0.472 \\
\hline \multicolumn{4}{|l|}{ Qualifying events } \\
\hline TIA & $134(78.8)$ & $12(100.0)$ & 0.052 \\
\hline Ischemic stroke & $36(21.2)$ & $0(0.0)$ & \\
\hline Treatment & & & 0.000 \\
\hline RMM & $53(31.2)$ & $10(83.3)$ & \\
\hline AMM & 117 (68.8) & $2(16.7)$ & \\
\hline
\end{tabular}

Data are expressed as numbers with percentages in parentheses unless otherwise indicated.

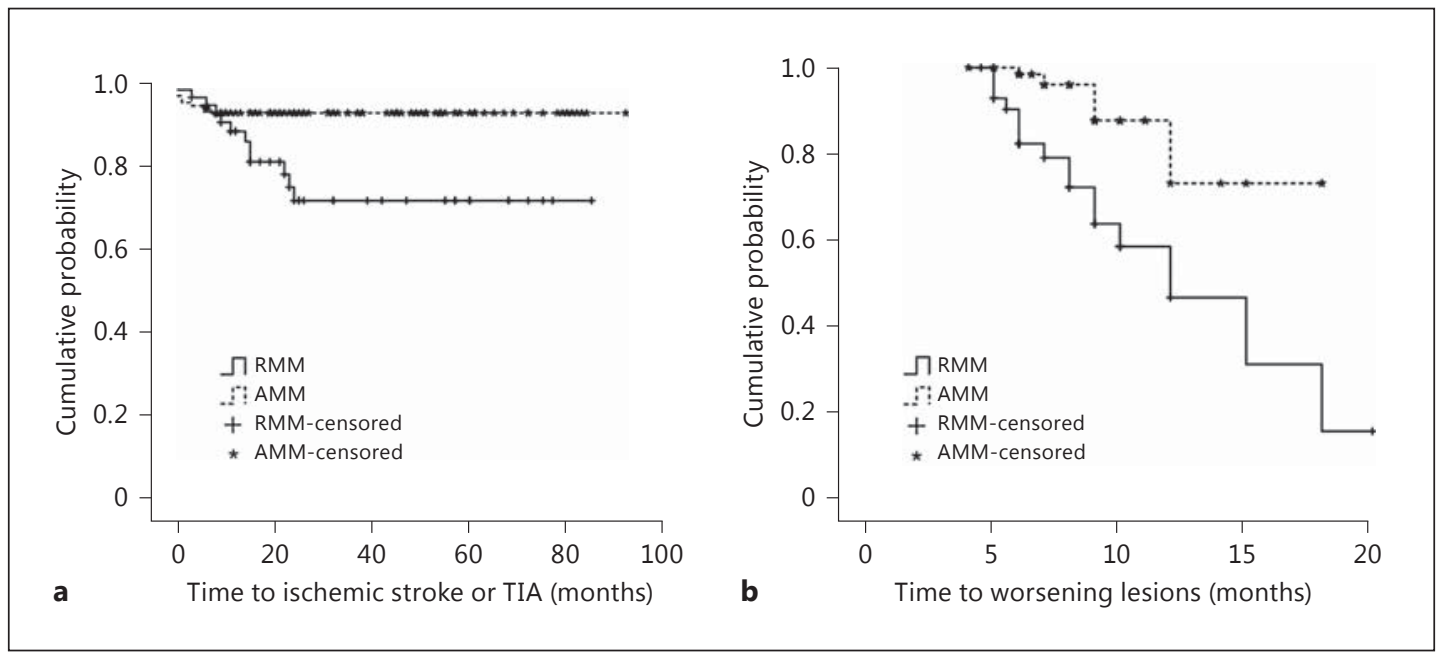

Fig. 1. Kaplan-Meier curves for cumulative incidence of neurological events (TIA or ischemic stroke; a) and cumulative worsening lesions (progressive original stenosis or new lesions; b).

without DSA follow-up were similar (table 1). Out of the 65 patients, 17 (15.4\%) were found to have 21 (13.2\%, 21/114; table 4) progressive stenoses and/or new lesions. Using KaplanMeier analysis, the cumulative 18-month progressive and/or new lesion-free rate was $38.2 \pm$ $1.45 \%: 15.6 \pm 1.34 \%$ in the RMM and $73.1 \pm 1.43 \%$ in the AMM group (log-rank test, $\chi^{2}=$ $11.513, \mathrm{p}=0.001$ ) (fig. 1b).

The baseline characteristics of the subsets of patients with and without recurrent stroke were further compared (table 3). Our data showed that the choice of drug treatment and the 
Liu et al.: Aggressive Medical Care in Young Chinese Patients with Ischemic Stroke of Undetermined Etiology: A Retrospective Study

Table 4. Lesion variables vs. risk of worsening lesions during angiographic follow-up

\begin{tabular}{lccc}
\hline Lesion characteristics & $\begin{array}{l}\text { Total } \\
(\mathrm{n}=114)\end{array}$ & $\begin{array}{l}\text { Worsening lesions } \\
(\mathrm{n}=21)^{\mathrm{a}}\end{array}$ & $\mathrm{p}$ \\
\hline Anterior circulation & $80(70.2)$ & $13(61.9)$ & 0.430 \\
$\quad$ Extracranial ICA & $24(21.1)$ & $4(19.0)$ & $5(4.8)$ \\
Intracranial ICA & $20(17.5)$ & $4(42.9)$ & \\
$\quad$ Middle cerebral artery & $34(29.8)$ & $0(0.0)$ & \\
$\quad$ Anterior cerebral artery & $2(1.8)$ & $0(38.1)$ & 0.614 \\
Posterior circulation & $34(29.8)$ & $7(28.6)$ & 0.000 \\
$\quad$ Posterior cerebral artery & $2(1.8)$ & $0(0.0)$ & 0.000 \\
$\quad$ Vertebral artery & $5(23.7)$ & $71.43 \pm 25.30$ & \\
$\quad$ Basilar artery & $73.59 \pm 20.26$ & $11(52.4)$ & \\
\hline Mean degree of stenosis \pm SD, $\%$ & $12(10.5)$ & $5(23.8)$ & \\
Recurrence & & & \\
Treatment & $43(37.7)$ & $(62.3)$ & \\
$\quad$ RMM & $71(2)$. & \\
AMM & & & \\
\hline
\end{tabular}

Data are expressed as numbers with percentages in parentheses unless otherwise indicated.

${ }^{\text {a }}$ Worsening lesions include progressive original lesions $(n=13)$ and new lesions $(n=8)$.

ESR were significantly associated with recurrent stroke (AMM vs. RMM: OR $=0.075,95 \% \mathrm{CI}$, 0.016-0.355, $\mathrm{p}=0.001$; ESR vs. no ESR: OR = 3.988, 95\% CI, 1.169-13.605, p = 0.027), whereas other variables were not. Using stepwise multivariate Cox regression analysis, AMM (crude $\mathrm{HR}=0.082,95 \% \mathrm{CI}, 0.018-0.375, \mathrm{p}=0.001$ ) was associated with a reduced risk of recurrent stroke and elevated serum levels of ESR (crude HR $=3.933$, 95\% CI, 1.247-12.404, p = 0.019) were associated with an increased risk of recurrent stroke. Furthermore, according to the $\chi^{2}$ test and multivariate Cox regression analysis, RMM was associated with worsening lesions including progressive or new arterial stenosis (AMM vs. RMM: OR $=0.128,95 \% \mathrm{CI}, 0.043-$ $0.384, \mathrm{p}=0.000$ ), and AMM was independently associated with a decreased risk of worsening lesions (crude HR $=0.211,95 \% \mathrm{CI}, 0.077-0.578, \mathrm{p}=0.002$ ). In addition, worsening lesions were the only independent predictor of recurrent stroke (after adjusted for all factors shown in table $3: \mathrm{HR}=35.000,95 \% \mathrm{CI}, 4.483-273.259, \mathrm{p}=0.001$ ).

Among the 65 patients ( 21 in the RMM and 44 in the AMM group) who had a DSA followup, only 17 (26.2\%) were detected to have worsening lesions including progressive original and new lesions (table 4). Among them, 5 were from the AMM group; however, only 2 patients presented with recurrent symptoms. Figure 2 shows typical angiograms of 3 cases before and after treatment with corticosteroids. The patients with progressive stenosis, new lesions, or clinical recurrence were advised to start azathioprine or cyclophosphamide therapy and had no recurrence of stroke (fig. 3a-c). A total of 12 patients in the RMM group had worsening lesions (fig. $3 \mathrm{~d}-\mathrm{f}$ ), and 3 received stent placement for progressive stenosis with recurrent stroke.

\section{Discussion}

In our study, we found no definite stroke risk factors in all participants aged 18-45 years, in spite of extensive clinical and laboratory investigations. Although some patients had abnormal serum levels of IgG, IgM, IgA, ESR, CRP, C3, or ASO, none of them met the diagnostic 
Fig. 2. a, b Imaging from a 37year-old man diagnosed with acute brainstem and cerebellar infarction. a Initial angiogram showing a total occlusion at the root of the right vertebral artery (arrow) and not detecting the distal vasculature. b After corticosteroid therapy for 6 months, the angiogram showed a 50\% stenosis in the proximal right vertebral artery (lower arrow) with good distal vasculature imaging (upper arrow). No clinical recurrence occurred during the 3-year followup. c, d Imaging from a 35-yearold man presenting with recurrent TIA in the anterior circulation. c Angiogram showing an $85 \%$ stenosis in the M1 segment of the right middle cerebral artery (arrow).d Angiogram showing no significantly changed lesion after treatment with corticosteroids for 6 months. However, the patient was asymptomatic.
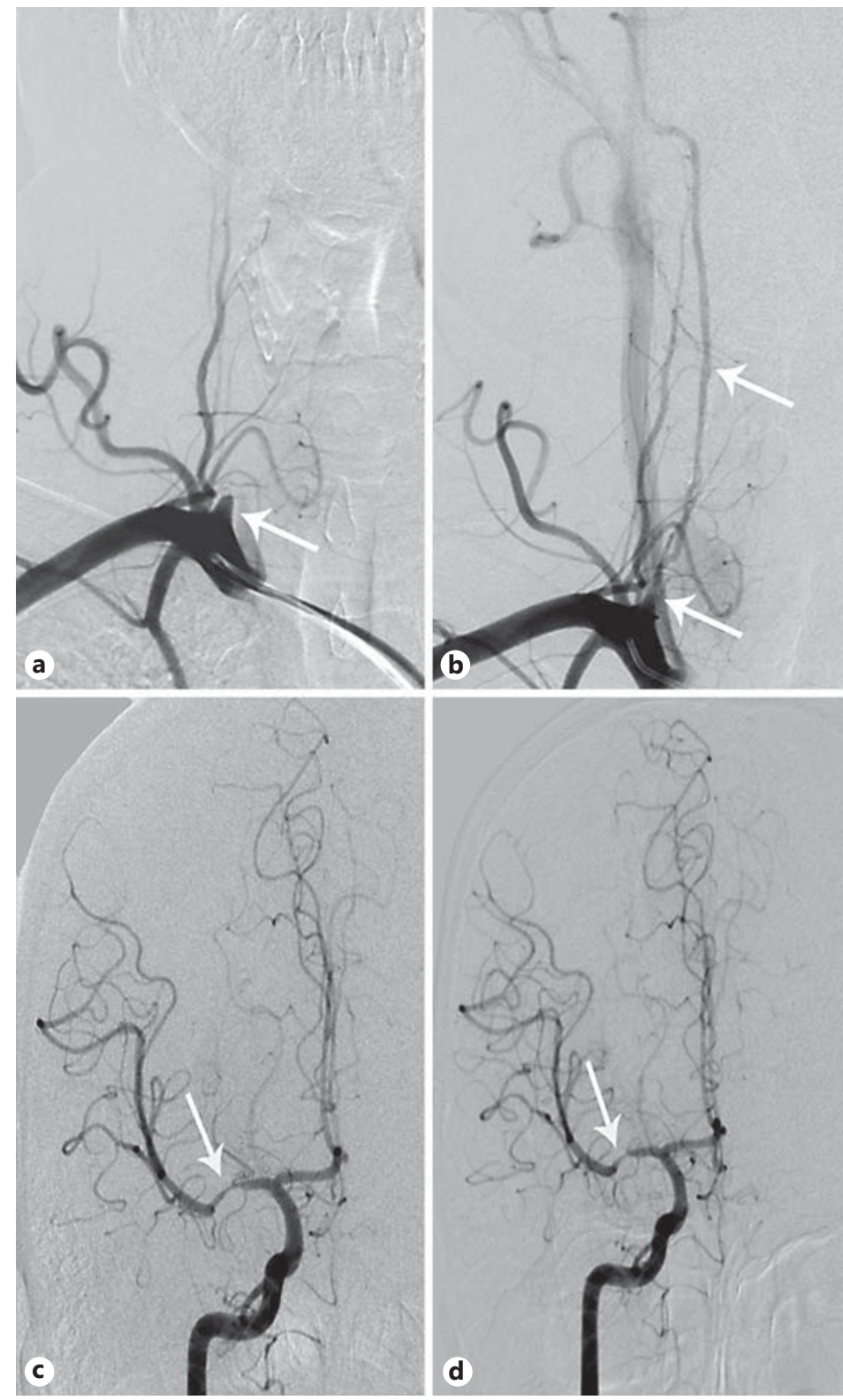

criteria of autoimmune system diseases such as systemic lupus erythematosus, Takayasu's disease, Behçet's disease, Sjögren's syndrome, rheumatoid arthritis, and primary central nervous system vasculitis, etc. In our study, the proportion of patients with strokes of undetermined etiology was $39.1 \%$, which is higher than that reported in a study from Taiwan (23.5\%) [10] and similar to that reported in other studies (31-62\% in young adult patients) $[3,11]$.

The proportion of male patients (74.9\%) was much higher than that in previous studies of cerebral infarction in young adults $[4,12]$. Except for ESR, there were no other differences between male and female patients. Furthermore, the frequency of lesions in the posterior circulation was lower and similar to that reported in other studies on young stroke patients $[1,13]$. More than $50 \%$ of the lesions in our study were located in the intracranial arteries, 
Liu et al.: Aggressive Medical Care in Young Chinese Patients with Ischemic Stroke of Undetermined Etiology: A Retrospective Study
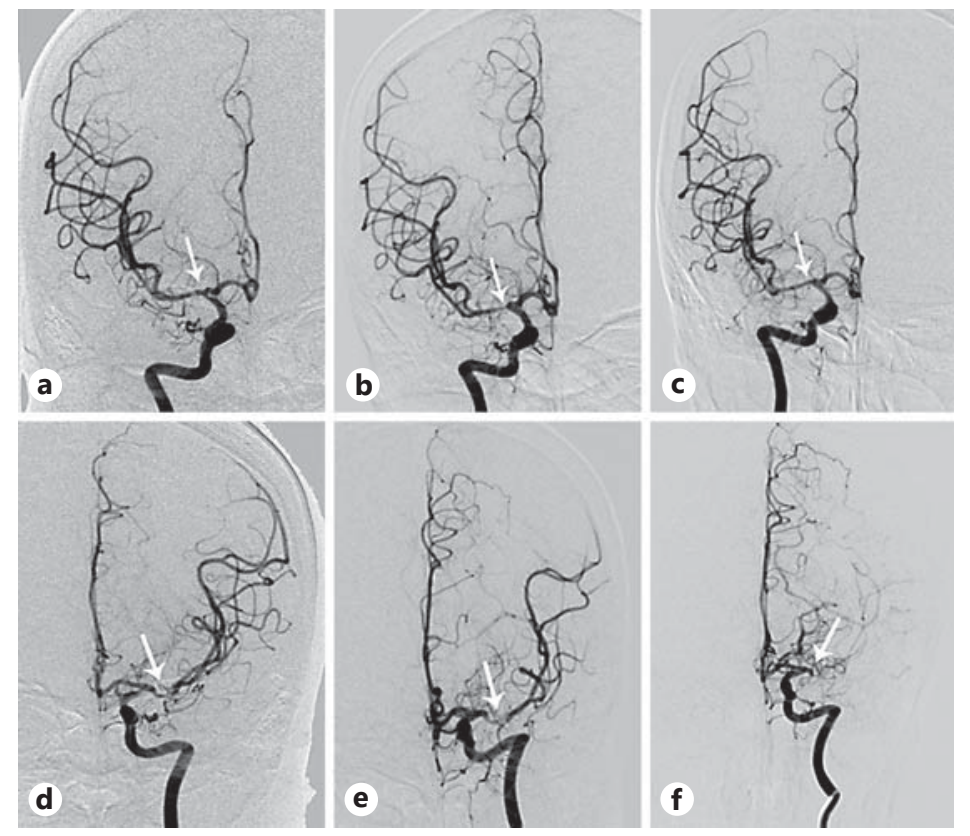

Fig. 3. a-c Imaging of a 39-year-old woman who presented with right cerebral infarction and who, in the process of corticosteroid reduction, developed recurrent TIA in the same vascular territory. Subsequently, she received azathioprine therapy, and no recurrence developed in the ensuing clinical follow-up. a Initial angiogram showing a mild stenosis at the root of the right middle cerebral artery (arrow). b Angiogram showing worsening original stenosis in the right middle cerebral artery after corticosteroid therapy for 4 months (arrow). c Angiogram showing no significant stenosis in the same artery after azathioprine therapy for 1.5 years. $\mathbf{d}$-f Imaging from a 33-year-old woman with frequent episodes of TIA in the distribution of the left middle cerebral artery who refused to receive corticosteroid therapy. $\mathbf{d}$ Initial angiogram showing a 95\% stenosis in the M1 segment of the left middle cerebral artery (arrow). e Angiogram showing a subtotal occlusion of the same lesion after RMM for 6 months (white arrow). $\mathbf{f}$ Angiogram showing a total occlusion of the left middle cerebral artery after 18 months (arrow).

which is consistent with previous reports showing that Asian patients have relatively higher occurrence rates of intracranial atherosclerotic lesions than White patients.

Despite the fact that $30 \%$ of all ischemic strokes have an unknown etiology, to date no information is available for special drug therapy and secondary prevention protocols for young patients with ischemic stroke of unknown etiology. Thus, these patients are usually treated according to guidelines that are applicable to patients with defined risk factors. According to the international and Chinese stroke association guidelines on ischemic stroke, all patients in the present study were provided a comprehensive treatment including lifestyle changes, risk factor management, antiplatelet therapy, and statin medication, regardless of the lipid levels. For the patients with symptomatic ICA stenosis $(\geq 50 \%)$, carotid endarterectomy or carotid angioplasty and stenting were recommended [14-16]. However, although intracranial stenting has been used in symptomatic patients with intracranial atherosclerotic stenosis in many neurological intervention centers, the results of the Stenting and Aggressive Medical Management for Preventing Recurrent Stroke in Intracranial Stenosis (SAMMPRIS) trial challenge this approach $[17,18]$. Furthermore, except for high complication and morbidity rates associated with percutaneous transluminal angioplasty and stenting, in-stent restenosis after intracranial stenting is an option which cannot be ignored. Some studies reported that in-stent restenosis tended to occur more often in young male patients $[19,20]$. In our 
cohort study, despite the fact that most patients had symptomatic ICA stenosis and/or intracranial atherosclerotic stenosis, all participants were young with undefined stroke risk factors and the majority of them were males. Therefore, endovascular interventions were not the first choice for them.

In view of the fact that some patients had abnormal serum levels of inflammation- or immunology-related parameters but did not meet the diagnostic criteria for vasculitis or autoimmune diseases, we presumed that inflammatory or immunological reactions might play a potential role in the course of vascular damage and underline the etiology of stroke. Based on this hypothesis, we attempted to treat some young stroke patients with corticosteroids (dexamethasone and/or prednisone) in accordance to the therapeutic protocol of vasculitis. In our study, the composite stroke recurrence rate was similar to that found in previous reports (3-15\%). Although the baseline data on admission between the RMM and AMM group were similar, during the mean 33 months of clinical follow-up, the patients in the AMM group had a significantly lower clinical recurrence rate (1.7 vs. $18.9 \%)$ as well as progressive and new lesion rate (5.6 vs. $1.4 \%$ and 20.9 vs. $16.3 \%$, respectively). Consistently, results from the Cox regression analysis showed that AMM could reduce the risks of clinical and angiographic end points, but the elevated serum levels of ESR might increase the risk of stroke recurrence. In addition, worsening lesion (progressive original or new arterial stenosis) was an independent predictor of recurrent stroke. In the AMM group, 2 patients experienced clinical recurrence and 5 had progressive artery stenosis. They received azathioprine therapy for 1 year no stroke occurred and the original lesions improved. Patients treated with corticosteroids did not undergo angioplasty or stenting, whereas 3 patients in the RMM group received endovascular interventions for aggravation of arterial stenosis and frequent recurrence of cerebral infarction and/or TIA. Since corticosteroids themselves have anti-inflammatory and suppressive immunological actions, our results suggest that inflammatory or immunological mechanisms did play a role in our patients, especially in those with elevated serum ESR.

This is a hospital-based direct comparison between young patients with ischemic stroke of unknown etiology treated with a routine and an aggressive protocol admitted to a single center. As a retrospective study with a relatively small sample size, there are several potential limitations. Firstly, angiographic follow-up was not available in all patients. In particular, fewer patients were reevaluated by DSA in the RMM group than in the AMM group, which may limit the statistical power and bias our results. Secondly, because treatment allocation between routine and corticosteroid therapy was not randomized, this may potentially influence the rate of progressive and/or new lesions or recurrent stroke. In the end, in view of the drawback of the retrospective study, some variables, such as the time interval between symptom onset and treatment and the characteristics of target lesions were not consistently or uniformly ascertained, which may influence patients' clinical outcomes or the angiographic outcomes of lesion arteries. This limitation may have led to an underestimation of asymptomatic events and other potential confounders in the comparison.

\section{Conclusion}

Based on our observation, we suggest that, although young patients with ischemic stroke may have more favorable long-term outcomes than elderly patients, when a young patient presents with ischemic stroke without risk factors - despite lifestyle changes, antiplatelet therapy, and statin therapy - corticosteroids may be recommended for preventing recurrent stroke and new arterial or worsening lesions. 
Liu et al.: Aggressive Medical Care in Young Chinese Patients with Ischemic Stroke of Undetermined Etiology: A Retrospective Study

\section{Acknowledgements}

This work was partly supported by grants from the National Natural Scientific Foundation of China (Nos. 81070923,81100870 and 81201078) and the Natural Scientific Foundation of Jiang Su Province (BK2011663).

\section{Disclosure Statement}

The authors declare that they have no competing interests.

\section{References}

1 Nakagawa K, Bianchi MT, Nakagawa SS, Sorond FA: Aggressive care after a massive stroke in young patients: is that what they want? Neurocrit Care 2010;13:118-122.

-2 Kwon SU, Kim JS, Lee JH, Lee MC: Ischemic stroke in Korean young adults. Acta Neurol Scand 2000;101:19-24.

- 3 Cerrato P, Grasso M, Imperiale D, Priano L, Baima C, Giraudo M, et al: Stroke in young patients: etiopathogenesis and risk factors in different age classes. Cerebrovasc Dis 2004;18:154-159.

4 Putaala J, Haapaniemi E, Metso AJ, Metso TM, Artto V, Kaste M, et al: Recurrent ischemic events in young adults after first-ever ischemic stroke. Ann Neurol 2010;68:661-671.

-5 Varona JF, Bermejo F, Guerra JM, Molina JA: Long-term prognosis of ischemic stroke in young adults. Study of 272 cases. J Neurol 2004;251:1507-1514.

6 Varona JF: Long-term prognosis of ischemic stroke in young adults. Stroke Res Treat 2010;2011879817.

7 Balci K, Utku U, Asil T, Celik Y: Ischemic stroke in young adults: risk factors, subtypes, and prognosis. Neurologist 2011;17:16-20.

8 Liu X, Xu G, Wu W, Zhang R, Yin Q, Zhu W: Subtypes and one-year survival of first-ever stroke in Chinese patients: The Nanjing Stroke Registry. Cerebrovasc Dis 2006;22:130-136.

-9 Zhu SG, Zhang RL, Liu WH, Yin Q, Zhou ZM, Zhu WS, et al: Predictive factors for in-stent restenosis after balloon-mounted stent placement for symptomatic intracranial atherosclerosis. Eur J Vasc Endovasc Surg 2010;40:499-506.

10 Lee TH, Hsu WC, Chen CJ, Chen ST: Etiologic study of young ischemic stroke in Taiwan. Stroke 2002;33:19501955.

11 Naess H, Nyland HI, Thomassen L, Aarseth J, Myhr KM: Etiology of and risk factors for cerebral infarction in young adults in western Norway: a population-based case-control study. Eur J Neurol 2004;11:25-30.

$\$ 12$ Fromm A, Waje-Andreassen U, Thomassen L, Naess H: Comparison between ischemic stroke patients $<50$ years and $\geq 50$ years admitted to a single centre: the Bergen stroke study. Stroke Res Treat 2011;2011:183256.

13 Putaala J, Metso AJ, Metso TM, Konkola N, Kraemer Y, Haapaniemi E, et al: Analysis of 1,008 consecutive patients aged 15 to 49 with first-ever ischemic stroke: the Helsinki young stroke registry. Stroke 2009;40: 1195-1203.

14 Lanzino G, Rabinstein AA, Brown RD Jr: Treatment of carotid artery stenosis: medical therapy, surgery, or stenting? Mayo Clin Proc 2009;84:362-387; quiz 67-68.

15 Lanzino G, Tallarita T, Rabinstein AA: Internal carotid artery stenosis: natural history and management. Semin Neurol 2010;30:518-527.

16 Patel TR, Bulsara KR: Current strategies for the treatment of intracranial atherosclerotic internal carotid artery stenosis. Neurosurg Rev 2009;32:23-27; discussion 27-28.

17 Al Hasan M, Murugan R: Stenting versus aggressive medical therapy for intracranial arterial stenosis: more harm than good. Crit Care 2012;16:310.

18 Chimowitz MI, Lynn MJ, Derdeyn CP, Turan TN, Fiorella D, Lane BF, et al: Stenting versus aggressive medical therapy for intracranial arterial stenosis. N Engl J Med 2011;365:993-1003.

19 Presbitero P, Carcagni A: Gender differences in the outcome of interventional cardiac procedures. Ital Heart J 2003; 4:522-527.

-20 Turk AS, Levy EI, Albuquerque FC, Pride GL Jr, Woo H, Welch BG, et al: Influence of patient age and stenosis location on wingspan in-stent restenosis. AJNR Am J Neuroradiol 2008;29:23-27. 